\title{
Highly conserved domains in hemagglutinin of influenza viruses characterizing dual receptor binding
}

\author{
Wei Hu
}

Department of Computer Science, Houghton College, Houghton, USA; wei.hu@,houghton.edu

Received 4 June 2010; revised 15 July 2010; accepted 18 July 2010.

\begin{abstract}
The hemagglutinin (HA) of influenza viruses initiates virus infection by binding receptors on host cells. Human influenza viruses preferentially bind to receptors with $\alpha 2,6$ linkages to galactose, avian viruses prefer receptors with $\alpha 2,3$ linkages to galactose, and swine viruses favor both types of receptors. The pandemic H1N1 2009 remains a global health concern in 2010. The novel 2009 H1N1 influenza virus has its genetic components from avian, human, and swine viruses. Its pandemic nature is characterized clearly by its dual binding to the $\alpha 2,3$ as well as $\alpha 2,6$ receptors, because the seasonal human $\mathrm{H} 1 \mathrm{~N} 1$ virus only binds to the $\alpha 2,6$ receptor. In previous studies, the informational spectrum method (ISM), a bioinformatics method, was applied to uncover highly conserved regions in the HA protein associated with the primary receptor binding preference in various subtypes. In the present study, we extended the previous work by discovering multiple domains in $\mathrm{HA}$ associated with the secondary receptor binding preference in various subtypes, thus characterizing the distinct dual binding nature of these viruses. The domains discovered in the HA proteins were mapped to the 3D homology model of HA, which could be utilized as therapeutic and diagnostic targets for the prevention and treatment of influenza infection.
\end{abstract}

Keywords: Binding Specificity; Discrete Fourier Transform; Electron-Ion Interaction Potential; Hemagglutinin; Influenza; Informational Spectrum Method

\section{INTRODUCTION}

Influenza A viruses have two surface proteins, hemagg- lutinin (HA) and neuraminidase (NA). HA is a homotrimer, in which each monomer comprises two subdomains, HA1 and HA2. HA1 initializes the contact with the cell membrane and HA2 mediates membrane fusion. In general, human influenza and avian viruses preferentially bind to the $\alpha 2,3$ sialylated and $\alpha 2,6$ sialylated glycan receptors, respectively [1-3]. Pigs have receptors for both human and avian influenza viruses on their tracheal cells, thus they can serve as a mixing vessel to re-assort genes from different species to make new influenza viruses. The 2009 H1N1 pandemic was caused by a novel swine-origin influenza A virus.

Using sequence analysis and homology modeling [4], the HA protein of $2009 \mathrm{H} 1 \mathrm{~N} 1$ was found to have the signature amino acid Asp190 and Asp225 known to confer binding affinity to $\alpha 2,6$ sialylated glycan receptors. The mutation Glu190Asp between avian and human H1 HA normally would lead to the loss of a critical contact with $\alpha 2,3$ glycans, which, however, was compensated by the presence of Lys145 in HA of 2009 H1N1. There were four loops in 2009 H1N1 HA, 130 loop, 140 loop, 150 loop, and 220 loop, each containing one Lys, to form a positively charged 'lysine fence' at the base of the binding site to support optimal contacts with the $\alpha 2,6$ and $\alpha 2,3$ receptors. Based on this structural analysis, it was predicted that the HA protein of $2009 \mathrm{H} 1 \mathrm{~N} 1$ virus can bind to the $\alpha 2,6$ as well as $\alpha 2,3$ sialylated glycan recaptors, which was subsequently verified by the carbohydrate microarray analysis in [5].

There were several other efforts in expanding the knowledge on 2009 H1N1. One study [6] investigated the three aspects of NA: the mutations and co-mutations, the stalk motifs, and the phylogenetic analysis. The potential mutations and strongly co-mutated positions of NA were found. A special NA stalk motif of high virulence, which was dominant in the past H5N1 strains, was discovered in H1N1 in 2009 for the first time. Another study [7] focused on HA and the interaction between HA and NA. The mutations of HA in 2009 H1N1 were found and mapped to the 3D homology model of H1, and the muta- 
tions on the five epitope regions on $\mathrm{H} 1$ were identified. The distinct response patterns of HA to the changes of NA stalk motifs were discovered, illustrating the functional dependence between HA and NA. With help from the results of the first study [6], two comutation networks were uncovered, one in HA and one in NA, where each mutation in one network co-mutates with the mutations in the other network across the two proteins HA and NA. These two networks residing in HA and NA separately might provide a functional linkage between the mutations that could change the drug binding sites in NA and those that could affect the host immune response or vaccine efficacy in HA.

The genes of 2009 H1N1 were derived from avian, human, and swine viruses. Identification of host shift markers of this novel virus remains an urgent and important research topic. However, sequence survey of 2009 H1N1 suggested the absence of the well-known host switch markers. A new procedure was designed to locate a collection of novel host markers in ten proteins/genes of 2009 H1N1 [8,9], which included, in addition to the SR polymorphism found in [10], a set of markers in PB2 that might play compensatory roles in the efficient replication and transmission of this new virus. These novel markers of 2009 H1N1 offered new and ample opportunities for further investigation of their biological functions in host adaptation to humans experimentally.

The informational spectrum method (ISM) [11] is a bioinformatics technique to study the biological functions of proteins with their physicochemical properties, which first translates a protein sequence into a numerical sequence based on each amino acid's electron-ion interaction potential (EIIP) and then the discrete Fourier transformation (DFT) is applied to it to create an informational spectrum. It is believed that the protein functions including the protein-protein interaction are encoded in the peaks of the informational spectrum.

In $[12,13]$ it was found that the CIS of HA1 of influenza strains have the primary characteristic dominant peaks at different IS frequencies as presented in Table 1. In this study, $F(0.295)$ will be referred to as pandemic human $\mathrm{H} 1 \mathrm{~N} 1$ receptor interaction frequency, $\mathrm{F}(0.055)$ as swine receptor interaction frequency, $F(0.076)$ as avian receptor interaction frequency, and $\mathrm{F}(0.236)$ as seasonal human $\mathrm{H} 1 \mathrm{~N} 1$ receptor interaction frequency. Some influenza strains display dual binding preference, one primary and one secondary, as demonstrated in $[12,13]$.

In $[12,13]$ the ISM was applied to investigate the interaction between HA protein and its receptors, which showed that HAlencodes highly conserved domains essential for receptor binding affinity. Their analysis also found the following receptor recognition domains in HA proteins from H1N1, H3N2, H5N1, and H7N7 (Table 2).
One region in HA1 of various strains was found to be connected with the primary receptor binding preference in [12] (Table 2). In [14], multiple such regions in HA1 responsible for the primary receptor binding preference in different subtypes were discovered (Table 3). In a study of receptor binding specificity of influenza viruses [14], the ISM was applied to find the mutations in HA1 and to elucidate the contribution to receptor binding switch from each mutation quantitatively. Two clusters of mutations in HA1 of 2009 H1N1 were uncovered, where the first was at residues 152-170 and the second at residues 257-278. The first cluster of mutations was contained in a pandemic human $\mathrm{H} 1 \mathrm{~N} 1$ receptor recognition domain (150:174) with the primary characteristic IS frequency at $\mathrm{F}(0.295)$ found in [15] (Table 3). Prompted by this finding, we searched for a similar domain in the vicinity of the second cluster, and found one such domain $(246: 286)$ associated with swine binding preference at the secondary characteristic IS frequency $F(0.055)$ [15] (Table 3). Our aim in this study was to extend these results by identifying multiple domains in HAl of influenza viruses associated with the secondary receptor binding preference, thus providing the complete information about the dual binding of the HA proteins. These conserved domains in HA1 might be used to develop targets for new drugs and infection control.

\section{MATERIALS AND METHODS}

\subsection{Sequence Data}

All HA sequences were retrieved from the Flu Resource

Table 1. Primary characteristic IS frequencies of HA proteins in $2009 \mathrm{H} 1 \mathrm{~N} 1$, swine H1N1/H1N2, avian H5N1, and seasonal human H1N1.

\begin{tabular}{ccccc}
\hline Subtype & 2009 & $\begin{array}{c}\text { Swine } \\
\text { H1N1 }\end{array}$ & $\begin{array}{c}\text { Avian } \\
\text { H1N2/H1N1 }\end{array}$ & $\begin{array}{c}\text { Seasonal human } \\
\text { H1N1 }\end{array}$ \\
\hline Frequency & $\mathrm{F}(0.295)$ & $\mathrm{F}(0.055)$ & $\mathrm{F}(0.076)$ & $\mathrm{F}(0.236)$ \\
\hline
\end{tabular}

Table 2. The receptor recognition domains of HA proteins in H1N1, H3N2, H5N1, and H7N7 influenza viruses.

\begin{tabular}{ccc}
\hline Strain & Frequency & Residues \\
\hline A/California/04/2009 (H1N1) & $\mathrm{F}(0.295)$ & $284-326$ \\
A/Hong Kong/213/03 (H5N1) & $\mathrm{F}(0.076)$ & $42-75$ \\
A/New Caledonia/20/99 (H1N1) & $\mathrm{F}(0.236)$ & $262-295$ \\
A/New York/383/2004 (H3N2) & $\mathrm{F}(0.363)$ & $57-90$ \\
A/equine/Prague/56 (H7N7) & $\mathrm{F}(0.285)$ & $28-61$ \\
A/Egypt/0636-NAMRU3/2007(H5N1) & $\mathrm{F}(0.236)$ & $99-132$ \\
A/South Carolina/1/18 (H1N1)) & $\mathrm{F}(0.258)$ & $87-120$ \\
\hline
\end{tabular}


Table 3. The receptor recognition domains of HA proteins in 2009 H1N1, swine H1N2, and avian H5N1 influenza viruses $[14,15]$.

\begin{tabular}{cccl}
\hline Subtype & Frequency & Residues & \multicolumn{1}{c}{ Consensus HA1 Sequence } \\
\hline 2009 H1N1 & $\mathrm{F}(0.295)$ & $106-130$ & SSVSSFERFEIFPKTSSWPNHDSNK \\
\hline 2009 H1N1 & $\mathrm{F}(0.295)$ & $150-174$ & WLVKKGNSYPKLSKSYINDKGKEVLV \\
\hline 2009 H1N1 & $\mathrm{F}(0.295)$ & $191-221$ & LYQNADAYVFVGSSRYSKKFKPEIAIRPKVR \\
\hline Swine H1N2 & $\mathrm{F}(0.055)$ & $1-29$ & DTLCIGYHANNSTDTVDTVLEKNVTVTHS \\
\hline Avian H5N1 & $\mathrm{F}(0.076)$ & $46-65$ & GVKPLILRDCSVAGWLLGNP \\
\hline Avian H5N1 & $\mathrm{F}(0.076)$ & $136-153$ & PYQGRSSFFRNVVWLIKK \\
\hline Avian H5N1 & $\mathrm{F}(0.076)$ & $269-286$ & LEYGNCNTKCQTPMGAIN \\
\hline
\end{tabular}

(http://www.ncbi/nlm.nih.giv/genomes/FLU/FLU.html) of the National Center for Biotechnology Information (NCBI) on Nov. 20, 2009. Only the full length and unique sequences were selected. There were $450 \mathrm{HA}$ sequences of human 2009 H1N1, 1228 HA sequences of avian H5N1 from 1959 to 2009, and 83 HA sequences of swine H1N2 from 1980 to 2009. All the sequences used in the study were aligned with MAFFT [16].

\subsection{Important Sites in HA}

Although there is a great variation due to high selection pressure in the HA1 sequences of various flu subtypes, the active site of HA1 is well conserved, which is located in a cleft composed of the residues 91, 150, 152, 180, 187, 191, and 192 [17]. The three amino acids at positions 187,191 and 192 are a part of the 190 helix. The active site cleft of HA is formed by its right edge (131 GVTAA) and left edge (221_RGQAGR) (H1 numbering), which are also commonly referred to as the 130 loop and 220 loop, respectively [18].

\subsection{Informational Spectrum Method}

The informational spectrum method is a bioinformatics technique that can be utilized to analyze protein sequences. Prior to this analysis, the protein sequences have to be translated into numerical sequences. One such approach is to assign each amino acid to its electron-ion interaction potential (EIIP), which represents the average energy of the valence electrons in the amino acid (Table 4).

Table 4. The electron-ion interaction potential (EIIP) of amino acids used to encode amino acids.

\begin{tabular}{cc|ccc}
\hline Amino acid & EIIP & & Amino acid & EIIP \\
\cline { 1 - 1 } L & 0.0000 & Y & 0.0516 \\
I & 0.0000 & W & 0.0548 \\
N & 0.0036 & Q & 0.0761 \\
G & 0.0050 & M & 0.0823 \\
E & 0.0057 & S & 0.0829 \\
V & 0.0058 & C & 0.0829 \\
P & 0.0198 & T & 0.0941 \\
H & 0.0242 & & F & 0.0946 \\
K & 0.0371 & R & 0.0959 \\
A & 0.0373 & D & 0.1263 \\
\hline
\end{tabular}

The application of EIIP to protein function analysis assumes that the strength of the electromagnetic field surrounding the protein is indicative of its biological function. This method was successful in revealing various protein properties [11].

The numerical sequence $x(m)$ of a protein sequence is transformed into the frequency domain using DFT. The DFT coefficients $X(n)$ are defined as

$$
X(n)=\sum x(m) e^{-j\left(\frac{2 \pi}{N}\right) n m} n=1,2, \ldots N / 2
$$

where $N$ is the length of sequence $x(m)$.

The energy density spectrum is defined as

$$
S(n)=X(n) X^{*}(n)=|X(n)|^{2}, n=1,2, \ldots, N / 2
$$

The informational spectrum (IS) of a sequence $x(m)$ comprises the frequencies and the amplitudes of its DFT.

Peak frequencies of IS of a protein sequence reflect its biological or biochemical functions. To determine the same biological or biochemical functions of a group of protein sequences, a consensus informational spectrum (CIS) can be used, which is defined as the product of energy density spectrum $S(n)$ of each sequence in the group. A measure of similarity for each peak is a signal-to-noise ratio $(\mathrm{S} / \mathrm{N})$, which is defined as a ratio of signal density to the mean value of the whole spectrum [11].

\subsection{Consensus HA1 Sequences}

We employed MAFFT to align the three consensus HA1 sequences of $2009 \mathrm{H} 1 \mathrm{~N} 1$, avian H5N1, and swine H1N2 (Figure 1). Each consensus sequence was then used in the ISM analysis to find the highly conserved domains in HA1 of different influenza subtypes.

\section{RESULTS}

As demonstrated in [12-15], the HA1 sequences in various influenza subtypes had a distinct inclination to interact with a specific primary receptor as well as a specific secondary receptor, and there were regions in HA1 encoding highly conserved information associated with the 


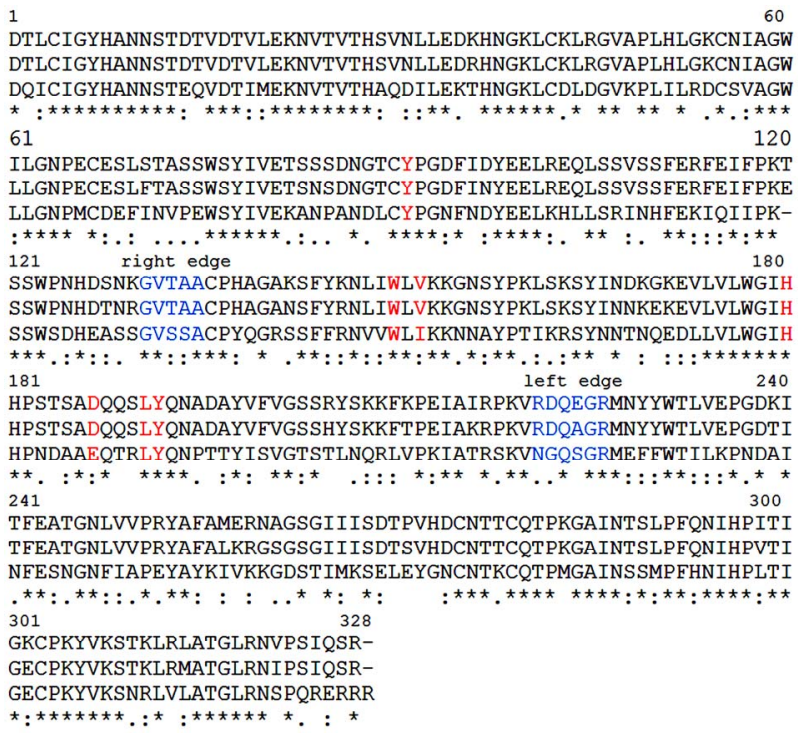

Figure 1. Multiple sequence alignment of three consensus HA1 sequences of $2009 \mathrm{H} 1 \mathrm{~N} 1$, swine H1N2, and avian H5N1 (presented in this order as well). The binding sites in HA are colored in red, the left and right edges of the binding cleft in blue.

primary binding preference. The main task of the present study is to explore the other parts of the HA1 sequences to find domains associated with the secondary binding preference. The entropy of HA1 of 2009 H1N1, swine H1N2, and avian H5N1 was calculated in [14], illustrating the most conserved positions in the HA1 sequences of each subtype. The ISM bioinformatics technique was applied to the three consensus HA1 sequences as presented in Figure 1 to uncover the conserved domains in HA1, which might affect the secondary receptor specificity in each subtype. In contrast, the ISM analysis in $[12,13]$ was applied to a particular selected strain in a subtype such as A/California/04/2009 in $2009 \mathrm{H} 1 \mathrm{~N} 1$ to find a conserved region. Overall, the conserved domains discovered by our approach using a consensus sequence had more coverage to different strains in a subtype than the single strain approach used in $[12,13]$.

\subsection{Conserved Domains in HA1 of 2009 H1N1}

Using the ISM, we discovered seven conserved domains in HA1 of 2009 H1N1 responsible for swine binding preference, which were located at residues 5-23, 63-81, $142-158,178-195,201-216,246-269$, and 277-287, respectively (Figure 2). The ISs of these domains and the consensus HA1 sequence in 2009 H1N1 were plotted in Figure 3. The IS of the consensus HA1 sequence in 2009 H1N1displayed a primary dominant peak of frequency $\mathrm{F}(0.295)$ and a secondary prominent peak of frequency $\mathrm{F}(0.055)$, demonstrating its dual binding preference. The domain 142:158 contained two binding sites 150 and 152
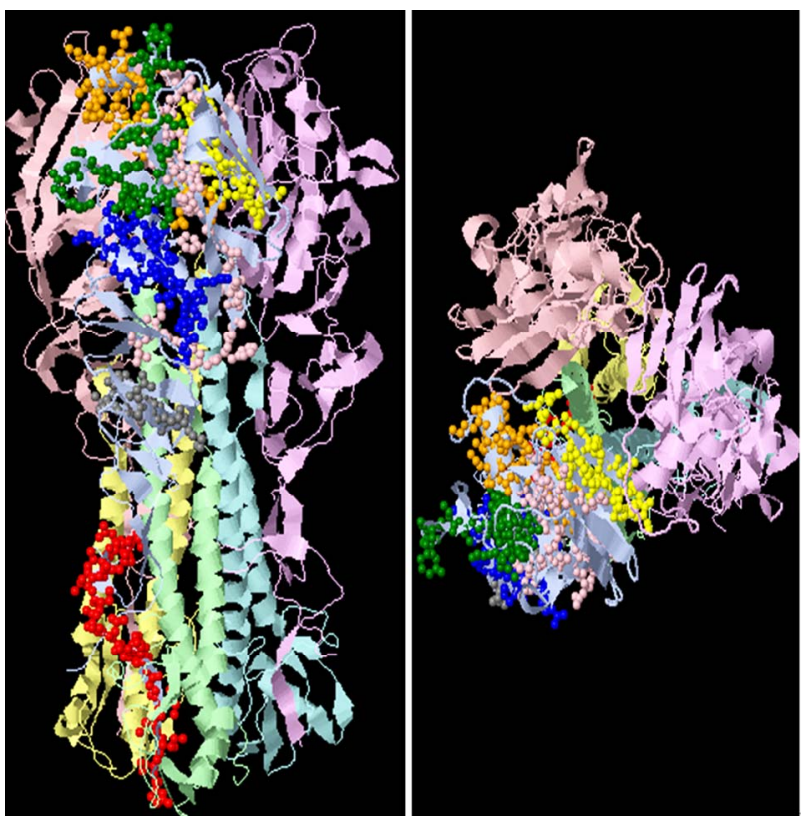

Figure 2. The two plots show in 3D structure the seven highly conserved domains related to swine binding characteristic found in HA of 2009 H1N1. Domain 5:23 is colored in red, domain 63:81 in blue, domain 142:158 in green, domain 178:195 in orange, domain 201:216 in yellow, domain 246:269 in pink, and domain 277:287 in gray (PDB code: 1RU7).

and was near the right edge of the active site. The domain 178:195 included four binding sites 180,187, 191, and 192. The domains $201: 216$ and 246:269 were close to the left edge of the active site, illustrating their important roles in receptor binding preference. As seen from the entropy distribution of HA1 of 2009 H1N1 in [14], these seven domains were highly conserved. In [12], a similar domain with human receptor binding characteristic was found in the C-terminus of the HA protein consisting of residues 284-326. Multiple such domains were identified in [14], and one domain with swine receptor binding characteristic was found in [15] (Table 1).

\subsection{Conserved Domains in HA1 of Swine H1N2}

In [12], the sequences of swine H1N1 and H1N2 were combined into a single dataset for analysis. Here the swine H1N2 was treated as a single dataset. The conserved domains found in this subtype were separated in two categories, i.e., one had swine binding preference (primary) and one had human binging preference (secondary). The four domains with swine binding were at residues 63-81, 178-189, 241-271, and 277-287, respectively (Figure 4), and the four domains with human binding were at residues 104-129, 189-213, 240-265, and 271-301, respectively (Figure 5). The ISs of these sequence in swine H1N2 revealed two dominant peaks at 
IS of consensus HA1 sequence of 2009 H1N1

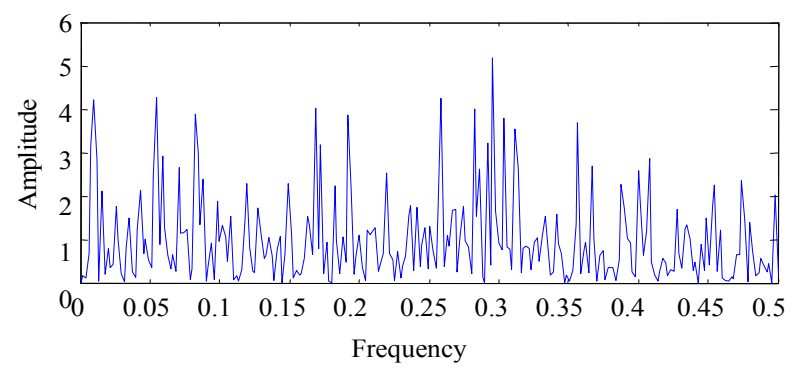

IS of a highly conserved domain (63:81) associated with

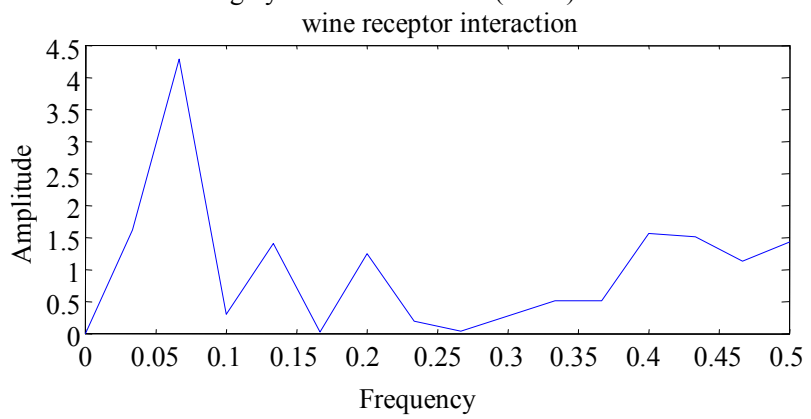

IS of a highly conserved domain (178:195) associated with swine receptor interaction

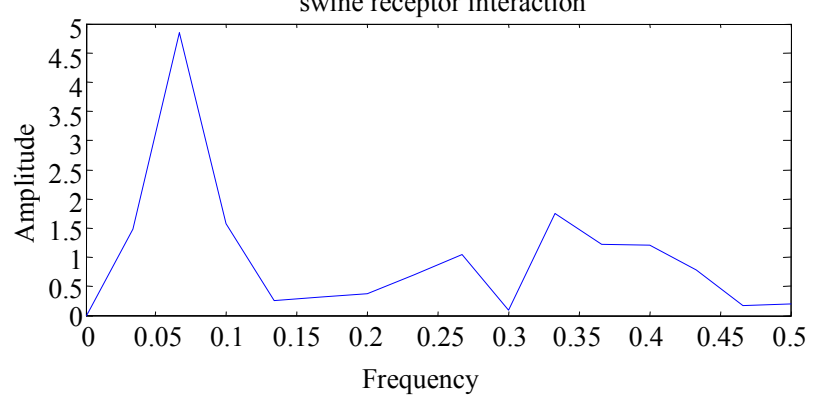

IS of a highly conserved domain (246:269) associated with

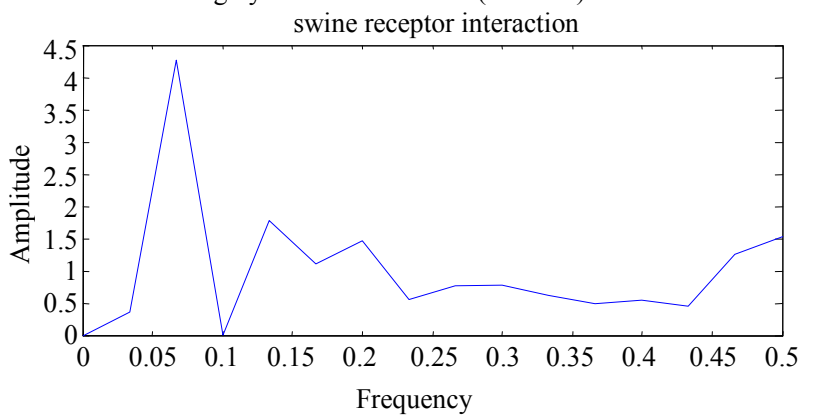

IS of a highly conserved domain $(5: 23)$ associated with

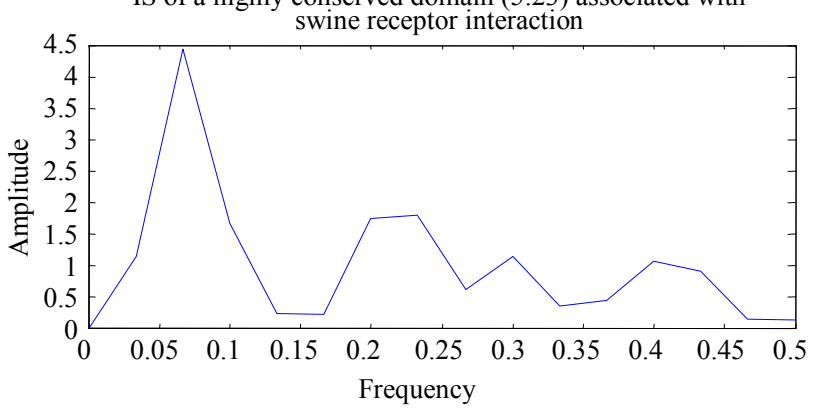

IS of a highly conserved domain (142:158) associated with

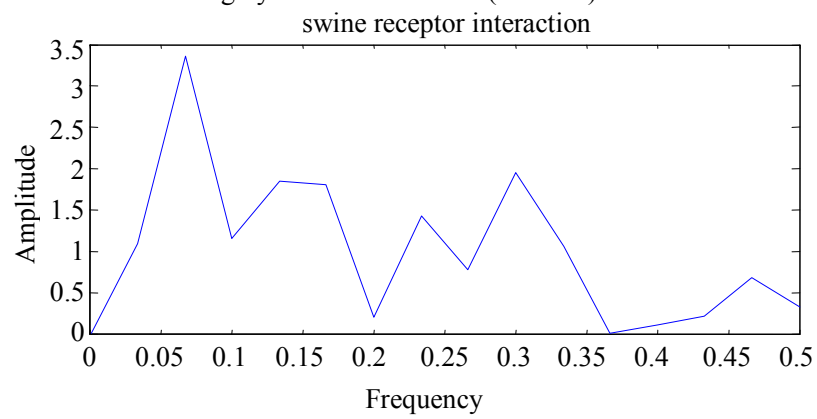

IS of a highly conserved domain (201:216) associated with swine receptor interaction

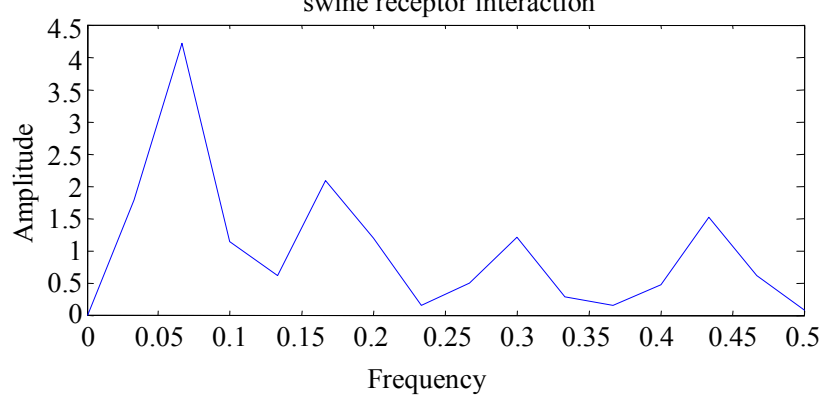

IS of a highly conserved domain (277:287) associated with swine receptor interaction

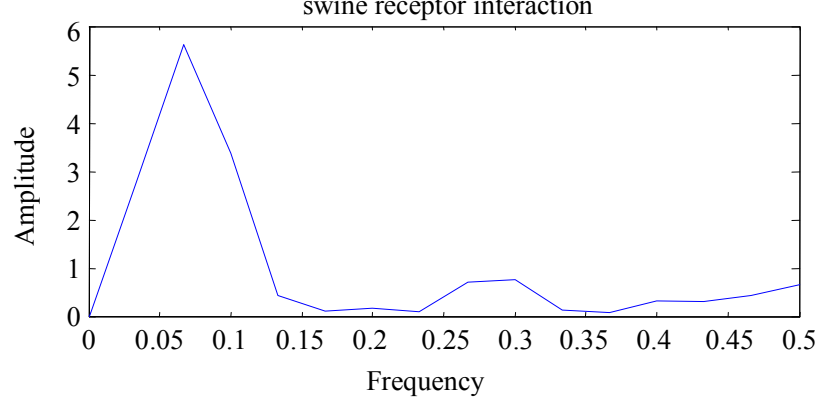

Figure 3. The eight plots show the informational spectrum of consensus HA1 sequence in 2009 H1N1 and that of the seven conserved domains in HA1 of 2009 H1N1 connected with swine receptor binding characteristic $(\mathrm{F}(0.055))$, respectively.

frequencies of $\mathrm{F}(0.055)$ (primary) and $\mathrm{F}(0.295)$ (seconddary). The entropy analysis in [13] suggested that these domains and the consensus HA1 sequence in swine H1N2 were displayed in Figure 6. The IS of the consensus HA1 domains were well conserved. One such domain in HA1 of swine H1N2 was located in the N-terminus of the protein at residues 1-29 [13] (Table 3).

\subsection{Conserved Domains in HA1 of Avian H5N1}

We found five conserved domains in HA1 of avian H5N1 relevant to human binding affinity, located at residues 9-39, 97-115, 123-139, 144-167, and 199-227, respectively (Figure 7). The ISs of these five domains and 

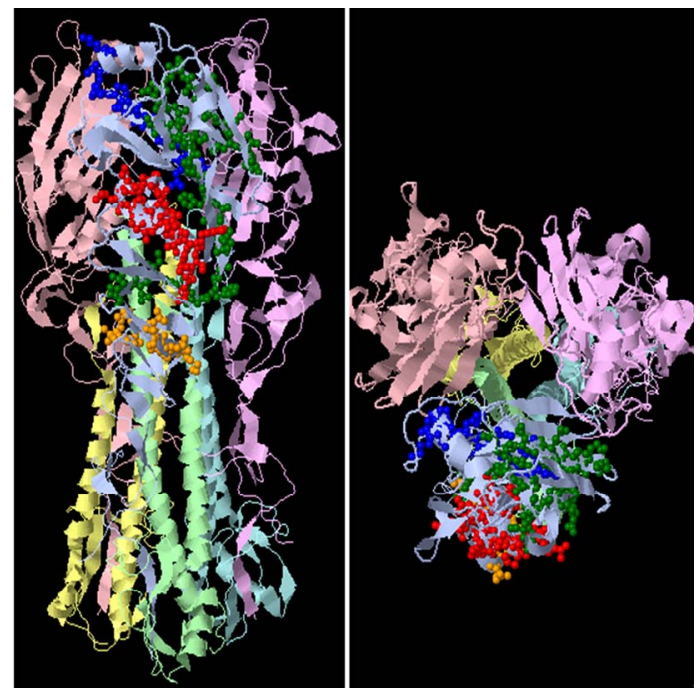

Figure 4. The two plots show in 3D structure the four highly conserved domains of swine binding characteristic found in HA of swine H1N2. Domain 63:81 is colored in red, domain 178:189 in blue, domain 241:271 in green, and domain 277:287 in orange.

IS of consensus HA1 sequence of swine H1N2

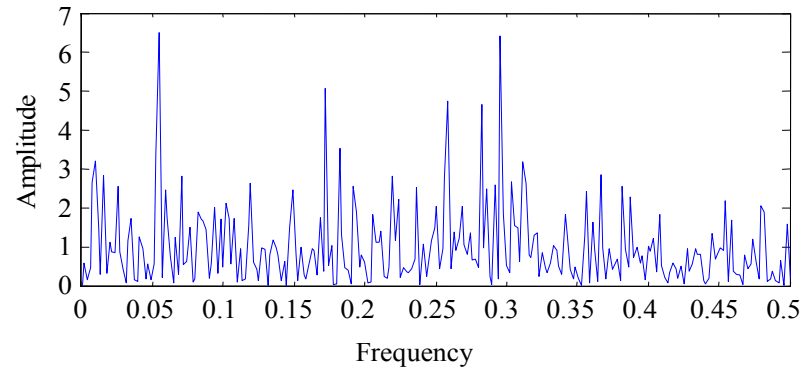

IS of a highly conserved domain (178:189) associated with swine receptor interaction

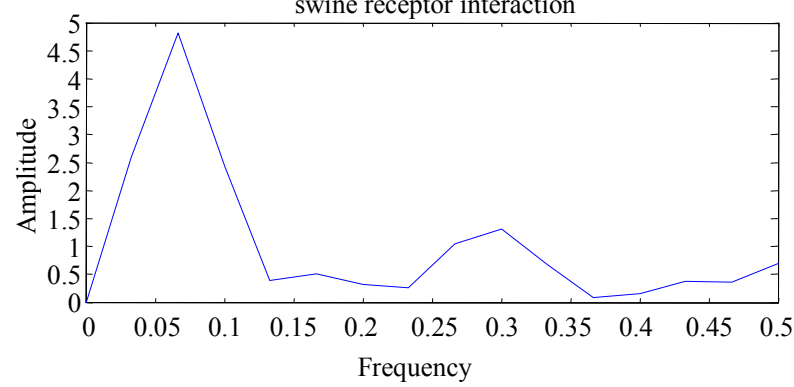

IS of a highly conserved domain (277:287) associated with swine receptor interaction

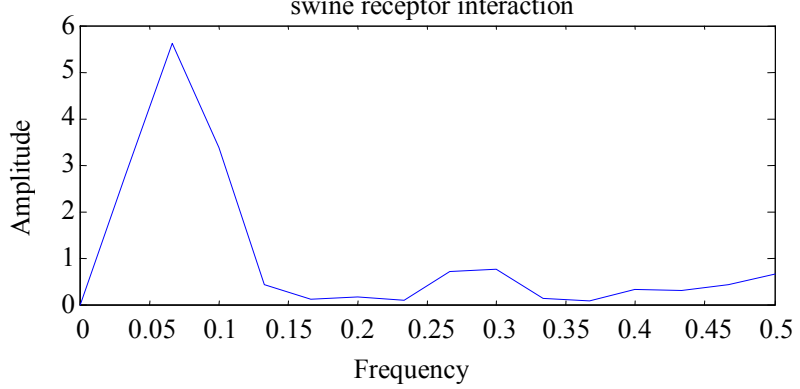

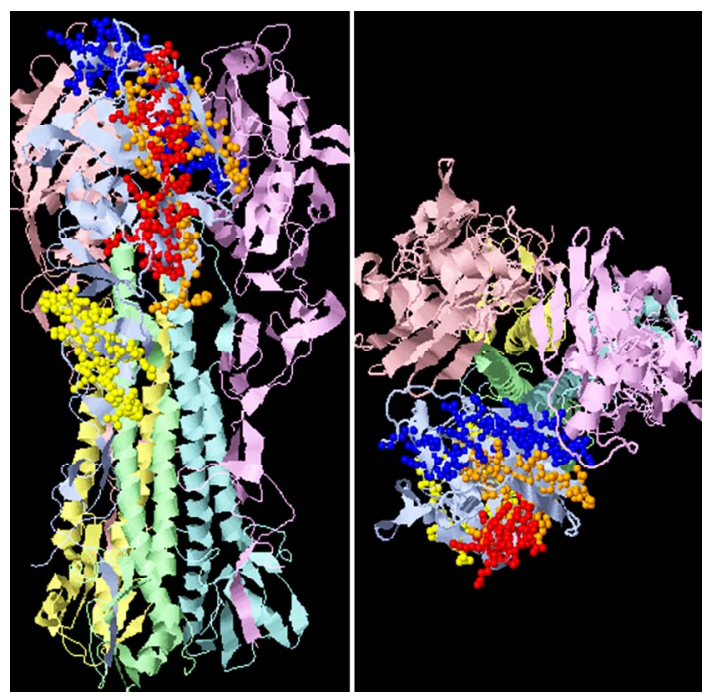

Figure 5. The two plots show in 3D structure the four highly conserved domains of human binding characteristic found in HA of swine H1N2. Domain 104:129 is colored in red, domain 189:213 in blue, domain 240:265 in orange, and domain 271:301 in yellow.

IS of a highly conserved domain (63:81) associated with swine receptor interaction

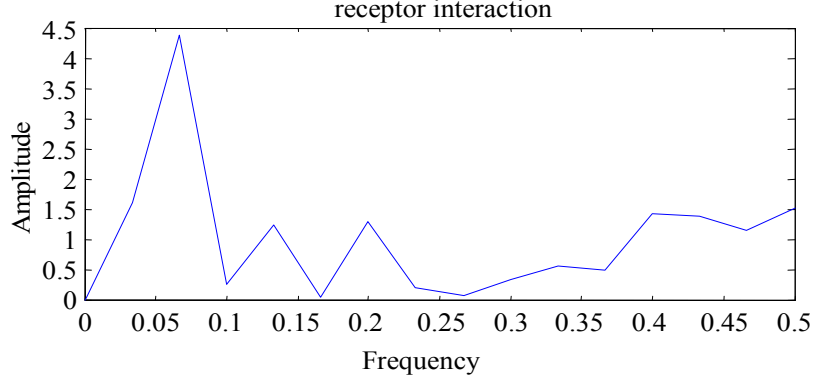

IS of a highly conserved domain (241:271) associated with swine receptor interaction

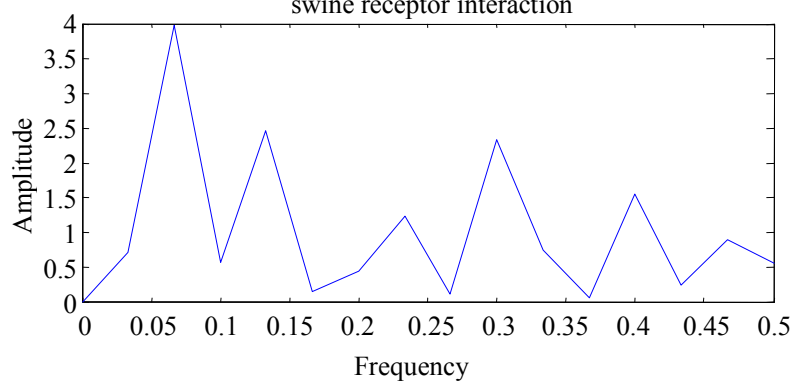

IS of a highly conserved domain (104:129) associated with pandemic human $\mathrm{H} 1 \mathrm{~N} 1$ receptor interaction

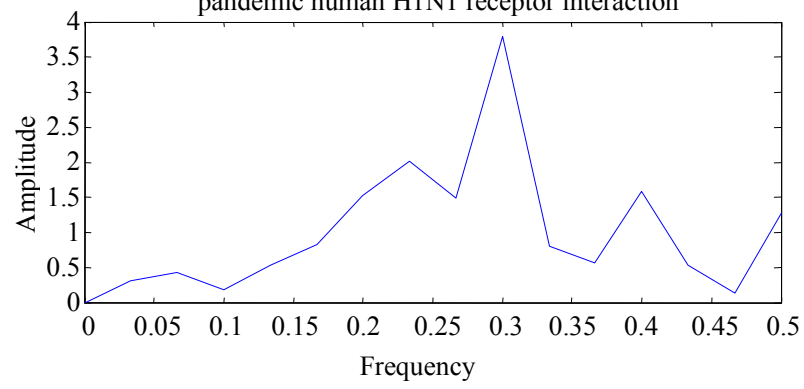



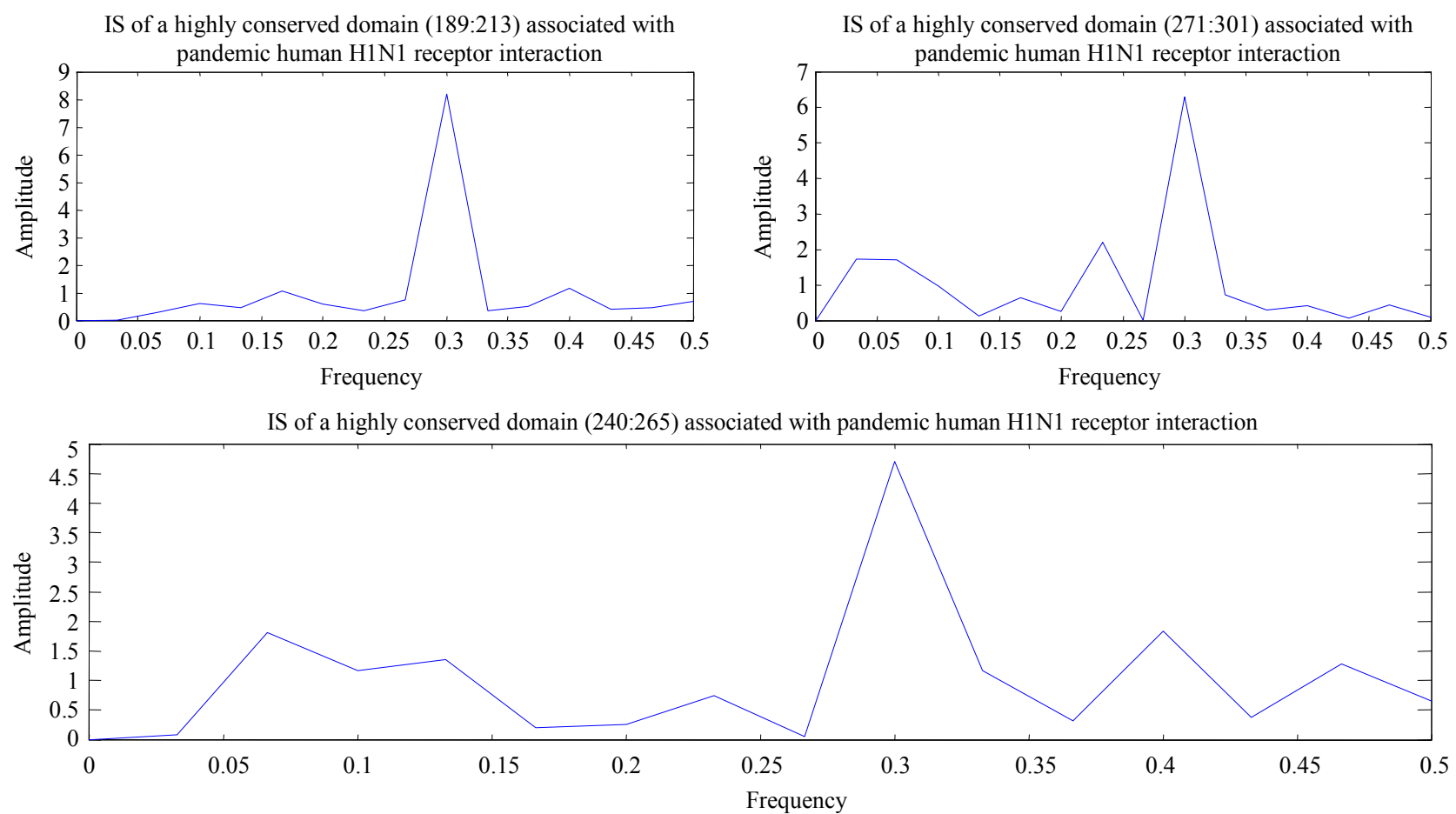

Figure 6. The nine plots show the informational spectrum of consensus HA1 sequence of swine H1N2, and that of seven conserved domains in swine H1N2 associated with human and swine binding $(\mathrm{F}(0.295)$ and $\mathrm{F}(0.055))$, respectively.
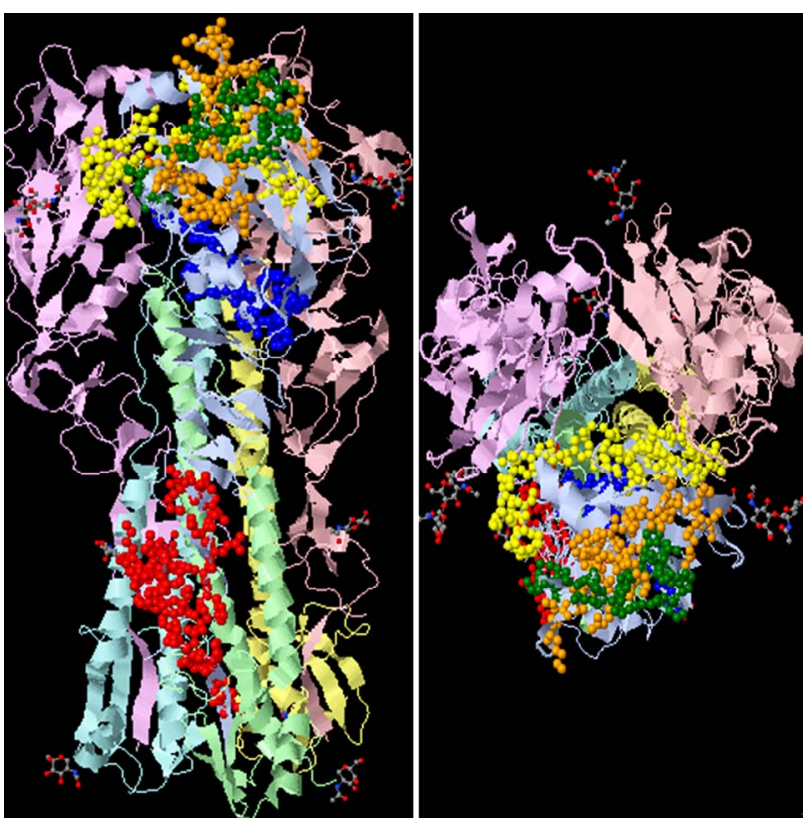

Figure 7. The two plots show in $3 \mathrm{D}$ structure the five highly conserved domains found in HA of avian H5N1 related to human binding. Domain 9:39 is colored in red, domain 97:115 in blue, domain 123:139 in green, domain 144:167 in orange, and domain 199:227 in yellow (PDB code: 2IBX).

the consensus HA1 sequence in avian H5N1 were illustrated in Figure 8. The IS of the consensus HA1 seque- nce in avian $\mathrm{H} 5 \mathrm{~N} 1$ demonstrated two dominant peaks of frequencies $\mathrm{F}(0.076)$ (primary) and $\mathrm{F}(0.236)$ (secondary). The entropy analysis in [14] implied that the five domains were well conserved, and the HA1 sequences of avian H5N1 were quite stable, in contrast to those of 2009 H1N1 and swine H1N2. In [13], a similar domain with avian binding was found in the N-terminus of the HA protein comprising residues 42-75. In [14], multiple such domains with avian binding were uncovered (Table 3).

\section{CONCLUSIONS}

Identifying the conserved characteristics of influenza viruses relevant to receptor binding preference is of great importance in flu research. The informational and structural properties of HAl of influenza viruses associated with the primary receptor affinity were investigated in [12-15]. To extend the previous results, we sought to uncover multiple domains in HA1 associated with the secondary binding preference, thus to expand our repertoire of these key regions in HA1 of influenza viruses.

The main findings of this study were presented in Table 5, which showed the locations, the characteristic IS frequencies, and the consensus sequences of the seven highly conserved domains in HA1 discovered in different subtypes. Combined with the results in Table 3 and those in $[12,13]$, these findings provided the complete 


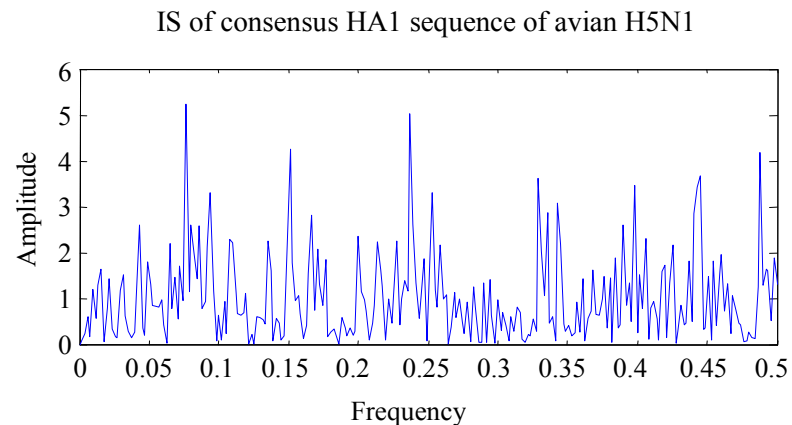

IS of a highly conserved domain (97:115) associated with

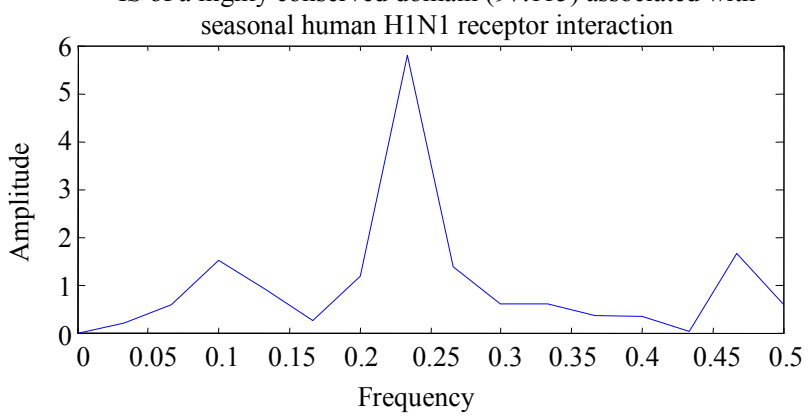

IS of a highly conserved domain (97:115) associated with

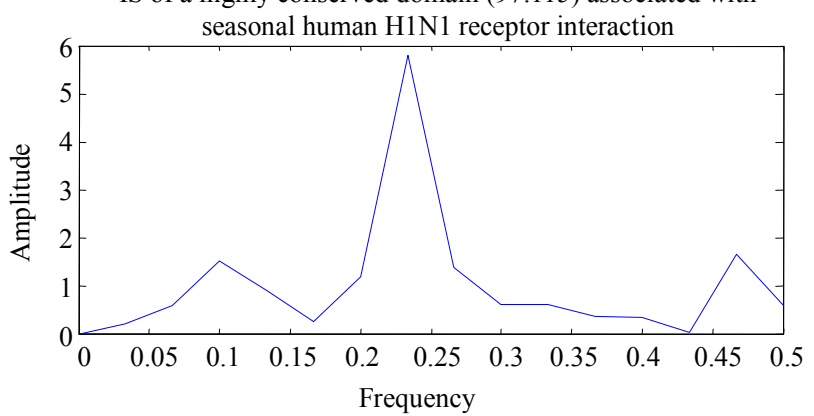

IS of a highly conserved domain (9:39) associated with

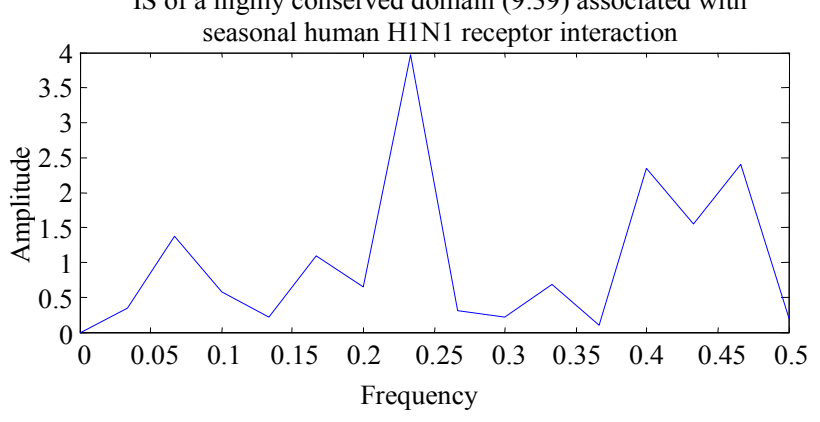

IS of a highly conserved domain (123:139) associated with seasonal human H1N1 receptor interaction

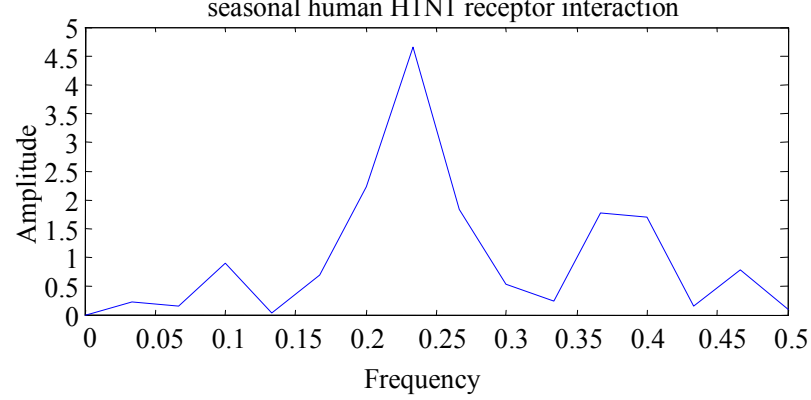

IS of a highly conserved domain (199:227) associated with

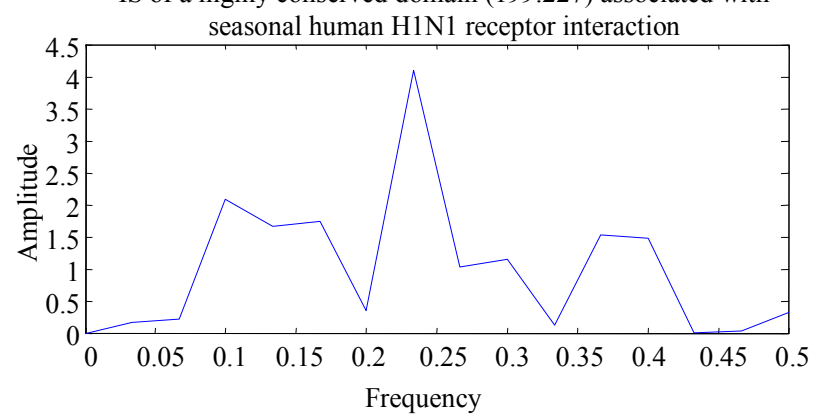

IS of a highly conserved domain (240:265) associated with pandemic human H1N1 receptor interaction

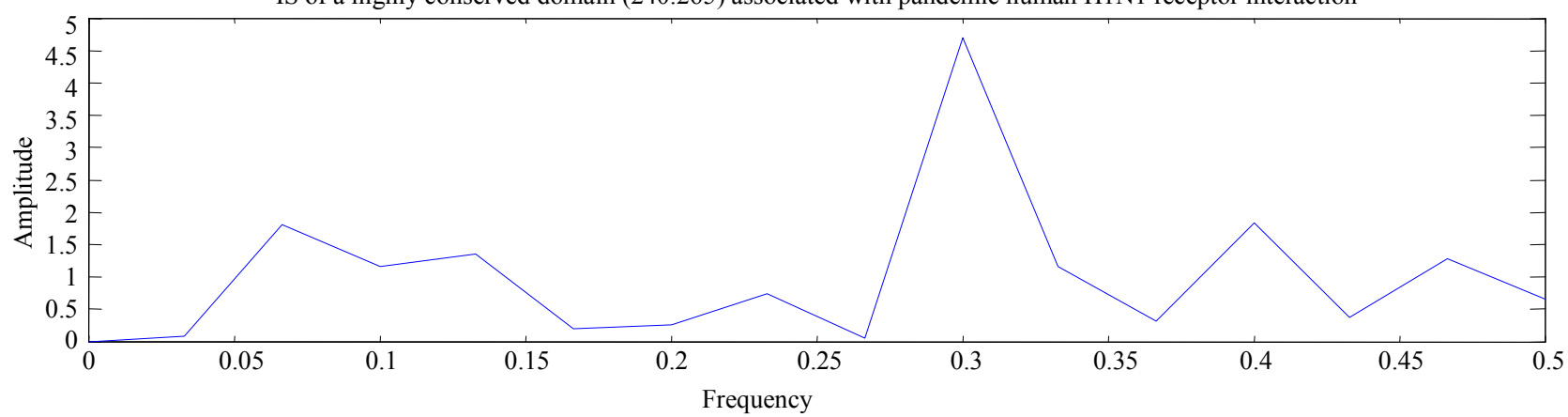

Figure 8. The seven plots show the informational spectrum of consensus HA1 sequence in avian H5N1 and the informational spectrum of the six conserved domains in avian $\mathrm{H} 5 \mathrm{~N} 1$ associated with human binding $(\mathrm{F}(0.236))$, respectively.

information about the conserved domains in HA1 that might modulate the dual receptor binding preference.

The domains in Table 5 are shorter than those discovered in $[12,13]$, implying that they were more easily conserved by the HA sequences than their longer counterparts. Furthermore, the identified multiple domains in HA1 related to dual receptor binding preference could provide ample options to design new therapeutic targets for drug development. Finally, because a consensus sequence of each subtype was employed to find these multiple domains in HA1, they are more representative of the strains in a given subtype than those obtained in $[12,13]$. 
Table 5. The receptor recognition domains in HA protein in 2009 H1N1, avian H5N1, and swine H1N2 influenza viruses.

\begin{tabular}{cccl}
\hline Subtype & Frequency & Residues & \multicolumn{1}{c}{ Consensus HA1 Sequence } \\
\hline 2009 H1N1 & $\mathrm{F}(0.055)$ & $5-23$ & IGYHANNSTDTVDTVLEKN \\
2009 H1N1 & $\mathrm{F}(0.055)$ & $63-81$ & GNPECESLSTASSWSYIVE \\
2009 H1N1 & $\mathrm{F}(0.055)$ & $142-158$ & KSFYKNLIWLVKKGNSY \\
2009 H1N1 & $\mathrm{F}(0.055)$ & $178-195$ & GIHHPSTSADQQSLYQNA \\
2009 H1N1 & $\mathrm{F}(0.055)$ & $201-216$ & VGSSRYSKKFKPEIAI \\
2009 H1N1 & $\mathrm{F}(0.055)$ & $246-269$ & GNLVVPRYAFAMERNAGSGIIISD \\
2009 H1N1 & $\mathrm{F}(0.055)$ & $277-287$ & TTCQTPKGAIN \\
Swine H1N2 & $\mathrm{F}(0.295)$ & $104-129$ & QLSSVSSFERFEIFPKESSWPNHDTN \\
Swine H1N2 & $\mathrm{F}(0.295)$ & $189-213$ & QSLYQNADAYVFVGSSHYSKKFTPE \\
Swine H1N2 & $\mathrm{F}(0.295)$ & $240-265$ & ITFEATGNLVVPRYAFALKRGSGSGI \\
Swine H1N2 & $\mathrm{F}(0.295)$ & $271-301$ & SVHDCNTTCQTPKGAINTSLPFQNIHPVTIG \\
Swine H1N2 & $\mathrm{F}(0.055)$ & $63-81$ & GNPECESLFTASSWSYIVE \\
Swine H1N2 & $\mathrm{F}(0.055)$ & $178-189$ & GIHHPSTSADQQ \\
Swine H1N2 & $\mathrm{F}(0.055)$ & $241-271$ & TFEATGNLVVPRYAFALKRGSGSGIIISDTS \\
Swine H1N2 & $\mathrm{F}(0.055)$ & $277-287$ & TTCQTPKGAIN \\
Avian H5N1 & $\mathrm{F}(0.236)$ & $9-39$ & ANNSTEQVDTIMEKNVTVTHAQDILEKTHNG \\
Avian H5N1 & $\mathrm{F}(0.236)$ & $97-115$ & DYEELKHLLSRINHFEKIQ \\
Avian H5N1 & $\mathrm{F}(0.236)$ & $123-139$ & SDHEASSGVSSACPYQG \\
Avian H5N1 & $\mathrm{F}(0.236)$ & $144-167$ & FRNVVWLIKKNNAYPTIKRSYNNT \\
Avian H5N1 & $\mathrm{F}(0.236)$ & $199-227$ & SVGTSTLNQRLVPKIATRSKVNGQSGRME \\
\hline
\end{tabular}

It was suggested in $[12,13]$ that the $2009 \mathrm{H} 1 \mathrm{~N} 1$ strains will continue to mutate in their HA gene, which could further favor the human interaction pattern by increasing the amplitude at frequency $F(0.295)$ and at the same time decreasing that at frequency $F(0.055)$. Identification of multiple domains in HA of influenza viruses related to dual receptor binding preference provided relevant information for the development of potential targets for new drugs and treatment of influenza infection

\section{ACKNOWLEDGEMENTS}

We thank Houghton College for its financial support.

\section{REFERENCES}

[1] Gambaryan, A., Tuzikov, A., Pazynina, G. et al. (2006) Evolution of the receptor binding phenotype of influenza A (H5) viruses. Virology, 344(2), 432-438.

[2] Iwata, T., Fukuzawa, K., Nakajima, K., et al. (2008) Theoretical analysis of binding specificity of influenza viral hemagglutinin to avian and human receptors based on the fragment molecular orbital method. Computational Biology and Chemistry, 32(3), 198-211.

[3] Yamada, S., Suzuki, Y., Suzuki, T., et al. (2006) Haemagglutinin mutations responsible for the binding of $\mathrm{H} 5 \mathrm{~N} 1$ influenza A viruses to human-type receptors. $\mathrm{Na}$ ture, 444(7117), 378-382.
[4] Soundararajan, V., Tharakaraman, K., et al. (2009) Extrapolating from sequence- the $2009 \mathrm{H} 1 \mathrm{~N} 1$ 'swine' influenza virus. Nature Biotechnology, 27(6), 510-513.

[5] Childs, R.A., Palma, A.S., Wharton, S., et al. (2009) Receptor-binding specificity of pandemic influenza A (H1N1) 2009 virus determined by carbohydrate microarray. Nature Biotechnology, 27(9), 797-799.

[6] Hu, W. (2009) Analysis of Correlated Mutations, Stalk Motifs, and Phylogenetic Relationship of the 2009 Influenza A Virus Neuraminidase Sequences. Journal of Biomedical Science and Engineering, 2(7), 550-558.

[7] Hu, W. (2010) The Interaction between the 2009 H1N1 Influenza A Hemagglutinin and Neuraminidase: Mutations, Co-mutations, and the NA Stalk Motifs. Journal of Biomedical Science and Engineering, 3(1), 1-12.

[8] Hu, W. (2010) Novel host markers in the 2009 pandemic $\mathrm{H} 1 \mathrm{~N} 1$ influenza A virus. Journal of Biomedical Science and Engineering, 3(6), 584-601.

[9] Hu, W. (2010) Nucleotide host markers in the influenza a viruses. Journal of Biomedical Science and Engineering, 3(7), 684-699.

[10] Mehle, A. and Doudna, J.A. (2009) Adaptive strategies of the influenza virus polymerase for replication in humans, Proceedings of the National Academy of Sciences of the United States of America, 106(50), 21312-21316.

[11] Cosic, I. (1997) The resonant recognition model of macromolecular bioreactivity, theory and application. Birkhauser Verlag, Berlin.

[12] Veljkovic, V., Niman, H.L., Glisic, S., et al. (2009) Identification of hemagglutinin structural domain and polymorphisms which may modulate swine H1N1 interactions with human receptor. BMC Structural Biology, 9, 
62.

[13] Veljkovic, V., Veljkovic, N., Muller, C.P., Müller, S., Glisic, S., Perovic, V. and Köhler, H. (2009) Characterization of conserved properties of hemagglutinin of $\mathrm{H} 5 \mathrm{~N} 1$ and human influenza viruses: possible consequences for therapy and infection control. BMC Structural Biology, 7, 9-21.

[14] $\mathrm{Hu}, \mathrm{W}$. (2010) Identification of highly conserved domains in hemagglutinin associated with the receptor binding specificity of influenza viruses: 2009 H1N1, avian H5N1, and swine H1N2. Journal of Biomedical Science and Engineering, 3(2), 114-123.

[15] Hu, W. (2010) Quantifying the effects of mutations on receptor binding specificity of influenza viruses. Journal of
Biomedical Science and Engineering, 3(3), 227-240.

[16] Katoh, K., Kuma, K., Toh, H. and Miyata, T. (2005) MAFFT version 5: improvement in accuracy of multiple sequence alignment. Nucleic Acids Research, 33(2), 511-518.

[17] KováccaronOVá, A., Ruttkay-Nedecký, G., HaverlíK, I.K., and Janecccaronek, S. (2002) Sequence Similarities and Evolutionary Relationships of Influenza Virus A Hemagglutinins. Virus Genes, 24(1), 57- 63.

[18] Gamblin, S.J., Haire, L.F., Russell, R.J., Stevens, D.J., Xiao, B., Ha, Y. et al. (2004) The Structure and Receptor Binding Properties of the 1918 Influenza Hemagglutinin. Science, 303(5665), 1838-1842. 